\title{
Aglossia: Case Report
}

\author{
Silvana Bommarito ${ }^{1}$ Luciana Escanoela Zanato ${ }^{2}$ Marilena Manno Vieira ${ }^{1}$ Fernanda Angelieri ${ }^{3}$ \\ ${ }^{1}$ Speach Therapy Department, Universidade Federal de São Paulo, São \\ Paulo, São Paulo, Brazil \\ 2 Human Health Department, Universidade Nove de Julho, São Paulo, \\ São Paulo, Brazil \\ Address for correspondence Silvana Bommarito, PhD, Departamento \\ de Fonoaudiologia, Universidade Federal de São Paulo, Rua Agostinho \\ Rodrigues Filho, 350 apto 152 A, São Paulo, SP 04026040, Brazil \\ (e-mail: bomarito@terra.com.br).
}

${ }^{3}$ Faculdade da Saúde, Universidade Metodista de São Paulo, São Paulo, São Bernardo do Campo, Brazil

Int Arch Otorhinolaryngol 2016;20:87-92.

\begin{abstract}
Keywords

- stomatognathic system

- tongue

- malocclusion

- articulation disorders

- deglutition disorders

Introduction Aglossia is a rare condition caused by failure of the tongue embryogenesis process (in the fourth to eighth weeks of gestation). The tongue is an organ used in different activities such as sucking, swallowing, chewing, and talking. It is also responsible for shaping palate dental arches (in its absence, they become atrophic). There are few similar cases reported in the literature.

Objective To describe a rare case of aglossia and the multidisciplinary professionals working together for 5 years to treat the patient.

Resumed Report An 8-year-old girl with aglossia had an assessment comprising: (1) clinical assessment of the stomatognathic system related to resting posture, tonus, and mobility; (2) orthodontic assessment; (3) surface electromyography of the chewing muscles; (4) swallowing videofluoroscopy.

Conclusion The authors confirmed the need of multidisciplinary cooperation to improve the patient's quality of life, because agenesia implicates many activities/ functions that depend on the tongue to fully work. Multiprofessional cooperation helps the patient learn compensation mechanisms.
\end{abstract}

\section{Introduction}

Aglossia is a rare condition caused by failure of the tongue embryogenesis process (in the fourth to eighth weeks of gestation). The tongue is the most mobile organ of the body and is associated with coordinated activities such as sucking, swallowing, chewing, and speaking, and it contributes to the normal development of the teeth and jaw. In the literature, few cases of aglossia are described and most are associated with genetic syndromes. The aim of this study is to describe an 8-year-old girl with complete aglossia and her treatment.

\section{Review of Literature with Differential Diagnosis}

The term aglossia refers to the congenital absence of the whole tongue. It is a rare condition, caused by a failure during the process of tongue formation. ${ }^{1}$ Embryogenic formation begins around the fourth week of intrauterine life, resulting in proliferation of the mesenchyme of the first pair of the gill arches. ${ }^{2}$ Most cases reported in the literature are associated with other manifestations and genetic syndromes, such as Moëbius and Pierre Robin. ${ }^{3,4}$ The craniofacial skeleton develops with predominantly vertical front and downward growth of the maxilla and mandible, compared with the skull base, complemented by breakout of the mandibular arches. Thus, morphological properties will adapt according to the central nervous system development to support new oral and craniomandibular structures with new joint surfaces breaking out to the muscles to develop occlusal forces. ${ }^{5}$ Therefore, the tongue is anatomically an organ belonging to the stomatognathic system with important participation in all functions: taste, sucking, swallow, chewing, and speech and articulation. It works mainly as an oral arch stabilizer and molder, and in received

October 31, 2014

accepted

December 16, 2014

published online

January 30, 2015
DOI http://dx.doi.org/

10.1055/s-0034-1544116. ISSN 1809-9777.
Copyright (c) 2016 by Thieme Publicações License terms Ltda, Rio de Janeiro, Brazil 


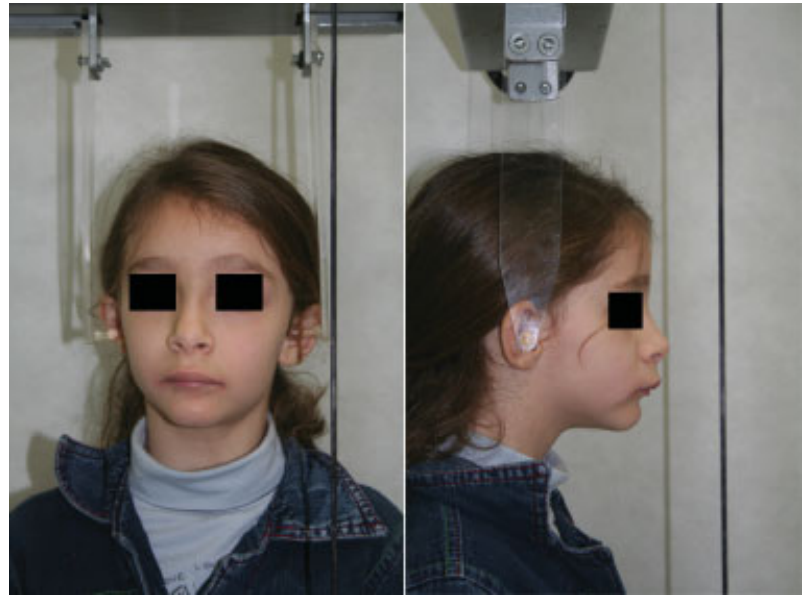

Fig. 1 (A) Frontal and (B) profile patient photos.

the tongue's absence, the mandibular dental arch becomes atresic. ${ }^{6,7}$ The first case of aglossia was reported in the eighteenth century, after which different cases were described in literature relating aglossia to important hand anomalies. ${ }^{4}$ Few cases were reported about aglossia not related to other alterations. However, this study, like two other studies, ${ }^{6,8}$ describes the case of isolated aglossia, because it was not possible to relate the case to some syndrome already described in the literature. In all reported cases, individuals showed alteration mainly in speech.

\section{Case Report}

The study was approved by the Research Ethic Committee CAAE:05167512.9.0000.5505. The patient (- Fig. 1A and 1B) presented for treatment in a university odontological clinic because of many carious lesions and consequent pain. Physical examination of the 8-year-old girl revealed the oropharynx (-Fig. 2A) and the complete absence of the tongue (-Fig. 2B). Medical history noted that the absence was congenital and not related to any other physical anomaly, except by otitis in early childhood. The mother also reported that the absence of the tongue was not observed in any other member of the family. Odontological treatment with repair and in some cases teeth extraction started by referring the patient to a speech therapist and orthodontic specialist for assessment, diagnosis, and therapy. The speech therapist noted phonoarticulatory structures in resting posture, lip tonus and mobility, cheek; mouth floor muscles, hard palate, soft palate, and uvula were observed ( - Fig. 2A). The patient showed competent lip seal during resting and lips showed normal tonus and mobility. During spontaneous talk, the patient showed extravagant lip movements, but muscular tonus was normal in two tests: (1) resistance against a spatula placed at the internal surface of the cheek after the order to compress them against examiner resistance and during their compression against the teeth (sucking) and (2) cheek filling with air test (inflation). During the physical exam, the patient showed an extremely deep hard palate, atretic maxillary and mandible dental arches, and absence of any palatal and/or submucosal cleft. The soft palate showed significant asymmetric mobility during sound emission and oblong uvula. Stomatognathic system functions were later assessed: breathing, sucking, chewing, swallowing, and speech.

\section{Breathing}

The patient showed mouth breathing throughout the assessment, which consisted of examining the presence of competent lip seal during resting posture associated with clinical assessment with the help of a millimetric mirror to check the air flow through the nostrils. ${ }^{9}$

\section{Sucking}

Sucking was assessed using a drinking straw and water. The patient was asked to suck through the drinking straw, and outstanding cheek movement, lip prehension, rhythm, and normal muscular force during the movement were observed.

\section{Chewing}

The patient was asked to chew a cream cracker. The child cut it in small pieces with her hands, introduced the pieces into the mouth cavity and chewed them with the help of exaggerated lip and cheek simultaneous and bilateral movements.

\section{Swallowing}

Swallowing was assessed with water and cream cracker. In water swallowing, compensatory movements of perioral muscles and the head movement to the back were observed. In cream cracker swallowing, the patient pushed the bolus with a finger to back of the mouth, in the direction of pharynx,

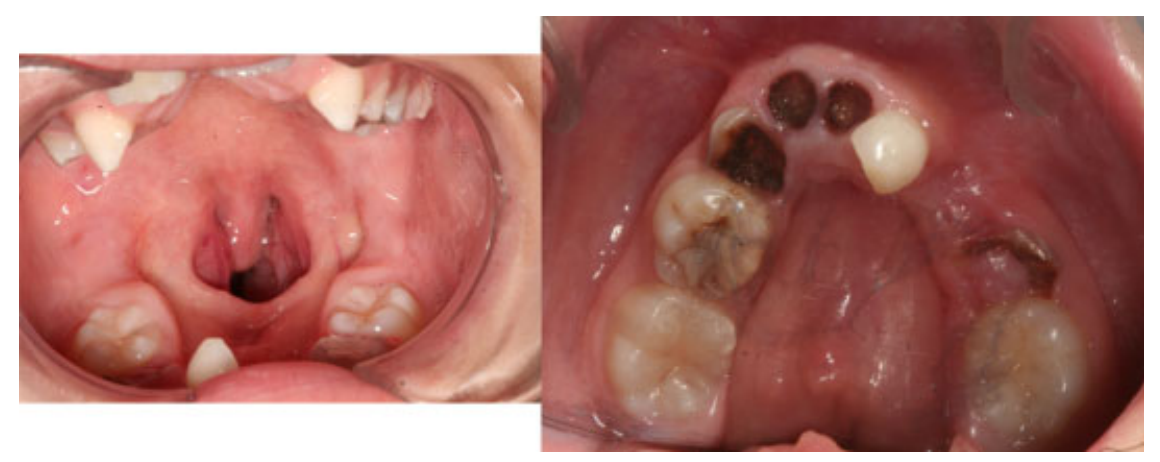

Fig. 2 Intraoral photos: (A) oral floor view and (B) mandibular dental arch. 
associated with head movement to the back, and she still needed water to swallow. Exaggerated perioral muscle movements to help swallowing were also observed.

\section{Speech}

The patient showed the following phoneme alterations: alveolar advance, liquid nasalization, plosive nasalization, and nasal speech. She uses the plosive $/ \mathrm{k} /, / \mathrm{g} /, / \mathrm{p} /$ and $/ \mathrm{b} /$; nasal $/ \mathrm{m} /$ and fricative $/ \mathrm{f} /$ and $/ \mathrm{v} /$. She also showed hypernasality. The case was assessed and monitored by the multiand interdisciplinary team, which found the following results after specific tests. Surface electromyography used eightchannel electromyographic equipment K6-I Diagnostic System (Myo-Tronics Research Inc., Kent WA, USA), which processes the electromyographic sign and converts the digital signals into analogical ones. It assessed bilaterally the temporal muscles and the masseter, digastric, and sternocleidomastoid in resting posture, with dental clenching at high-level strength, either chewing or swallowing the cream cracker. There was no sign of hyper- or underactivity independent of the ingested food or assessed activity.

Swallowing videofluoroscopy was performed in a specialized center by a speech therapist. The patient was instructed to swallow liquid (water), pasty food (creamy yogurt), and a solid food (wafer cracker), all mixed with pure barium to produce contrast. The patient presented functional swallowing with low stasis in the posterior pharyngeal wall. In addition, there was pronounced elevation of the oral cavity floor and important lowering of the palatine velum with great constriction of the oropharyngeal muscle as if the mouth floor was a "tongue."

Orthodontic evaluation consisted of clinical assessment with complementary exams making complete orthodontic documentation with extra- and intraoral photos, study models, teleradiography, radiographies of anterior teeth, and panoramic X-rays. Computed tomography (cone beam) and facial analysis also complemented diagnosis. In extrabuccal assessment, the patient presented facial asymmetry and absence of competent lip seal, described by the interposition of the lower lip between the anterior maxillary and lower teeth.

In lateral view (-Fig. $\mathbf{3 A}$ and $\mathbf{3 B}$ ), the facial profile was strongly convex, with the lower lip slightly subverted and regular nasolabial angle. - Fig. $3 C$ shows a frontal view, with narrowing of the upper and lower arch. In intraoral view of the oral cavity and oropharynx, the absence of tongue could be seen ( $\mathbf{F i g . ~ 2 A )}$ ). Intraoral clinical assessment revealed that the patient had mixed dentition in the first transitory period, when permanent incisor teeth are in eruption phase associated with the first permanent molars. Many teeth had dental caries, mainly the deciduous ones: first right maxillary molar, second right lower molar, first left lower molar, and two central lower incisors, which can be observed in -Fig. 2B. She had early loss of left lower canine, lower lateral incisors, first right lower molar, and also the deciduous teeth. High space loss was evident in the lower dental arch. This medical condition is more concerning when related to the fact that both maxillary and mandibular dental arches had high-level atresia (-Fig. 3A, 3B, and $\mathbf{3 C}$ ). Also, the increase in the horizontal distance between the anterior maxillary and lower teeth created a space in which the lip was placed either in resting posture or in functions of the stomatognathic system (-Fig. 3B).

-Fig. 4A shows the panoramic X-ray depicting the absence of mandibular lateral incisors and one mandibular central incisor. Furthermore, the patient was in the period of mixed dentition suitable to her chronological age, following the regular sequence of permanent teeth replacement, also observed in panoramic X-ray. The lateral cephalometric radiography ( - Fig. 4B) and cone beam computed tomography ( - Fig.5) revealed that the maxilla and mandible were regular size in the sagittal view and were related to each other, also shown in -Figs. 6A, B, C and 7A, B, C. On the other hand, high retrusion of the lower dental arch was evident. This fact, together the high tooth inclination to buccal surface of maxillary incisors (-Fig. 6A), provided a very accentuated overjet. Transversely, high maxillary atresia was observed mainly in the mandible (-Fig. 7B). Absence (agenesia) of three permanent lower incisors was also registered.

\section{Discussion}

Aglossia is a rare condition described in the literature. ${ }^{2-6,8}$ The tongue plays an important role in the growth of the maxillomandibular complex, which includes the medial and inferior third of the face. As the function is completely related to shape, all stomatognathic system functions are implicated in the sense that the bigger the deformity, the bigger the functional damage.

Good positioning and balance of the lips and buccinator muscle are essential for adequate sagittal mandible growth. ${ }^{10}$ In this case, the sagittal growth of mandible was comparable to the sagittal growth of the maxilla, which leads to the hypothesis that the tongue function is to mold the maxillary and lower dental arches (dental-alveolar processes).The need for space for teeth eruption is a necessary stimulus for their growth. In the present patient, the absence of three inferior dental germs (two lateral incisors and one central incisor) was registered. This fact associated with the absence of the tongue caused reduced growth of the inferior dental arch.

Differences between the superior and inferior dental arches produced an increase of distance between the superior and inferior teeth, leading to the interposition of inferior lip in the resting situation as well as during activity, ${ }^{11}$ thus aggravating the problem. The higher the strength of the inferior lip, the bigger the deformity. This atresia may be confirmed by the $30-\mathrm{mm}$ transpalatine distance compared with the normal 34-mm value.

In the inferior dental arch, the distance between the inferior molars (measured by tongue surface) was $18 \mathrm{~mm}$. However, the absence of the tongue had a fundamental importance in the transversal growth of the mandible and maxilla. This fact contributed to the absence of space available for permanent teeth eruption mainly in the inferior dental arch, which was worsened by the premature loss of the inferior deciduous teeth. Absence of the tongue 

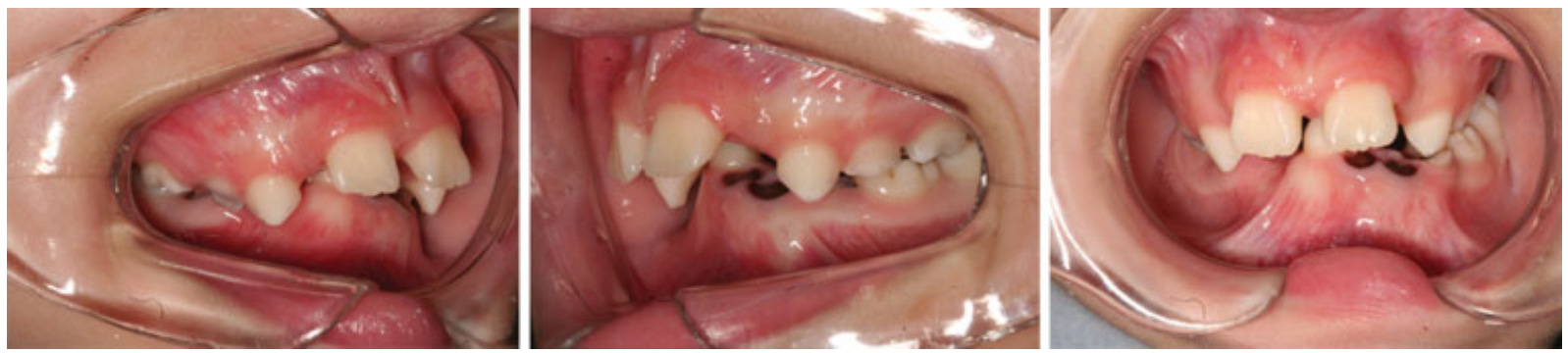

Fig. 3 Intraoral photos: (A) right side; (B) left side; (C) frontal views.

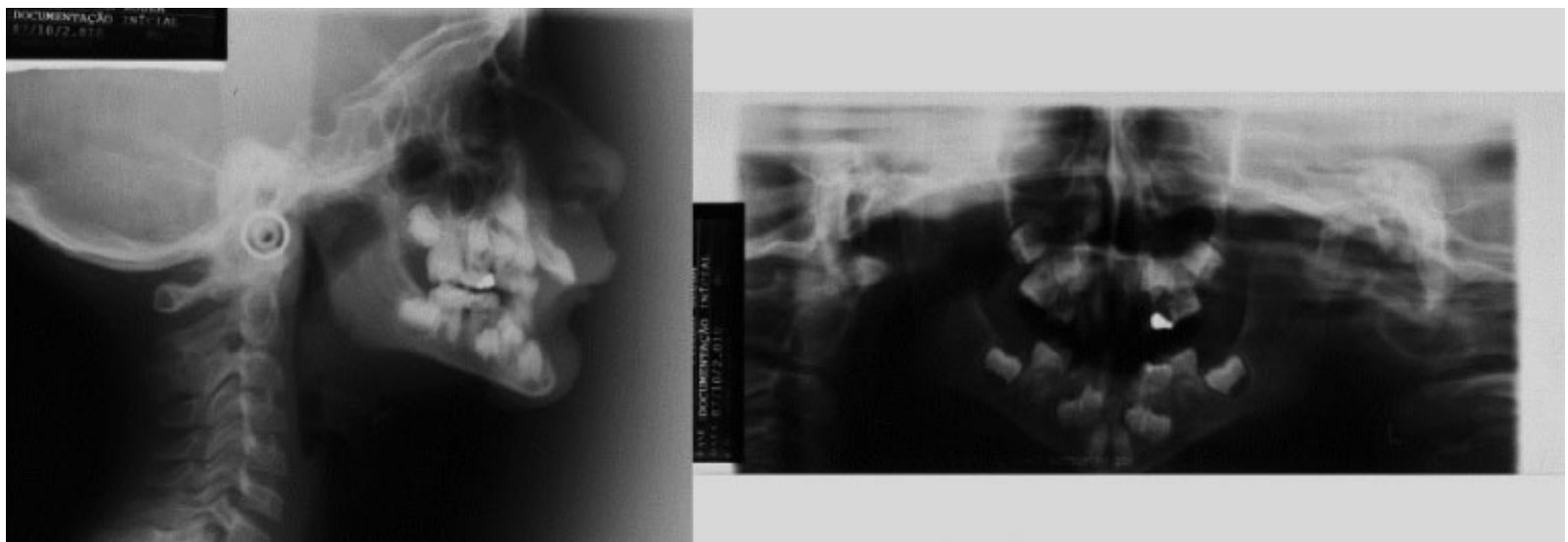

Fig. 4 (A) Normal lateral teleradiography and (B) panoramic radiography.
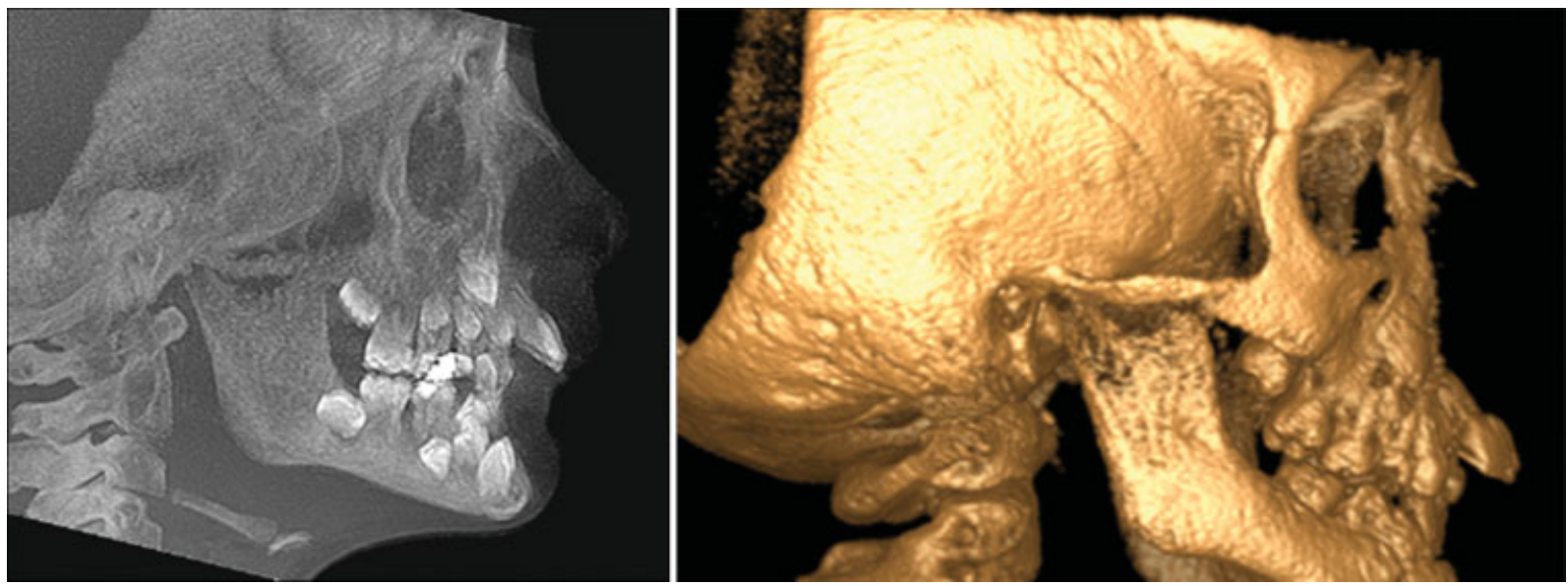

Fig. 5 Computed tomography in profile view.
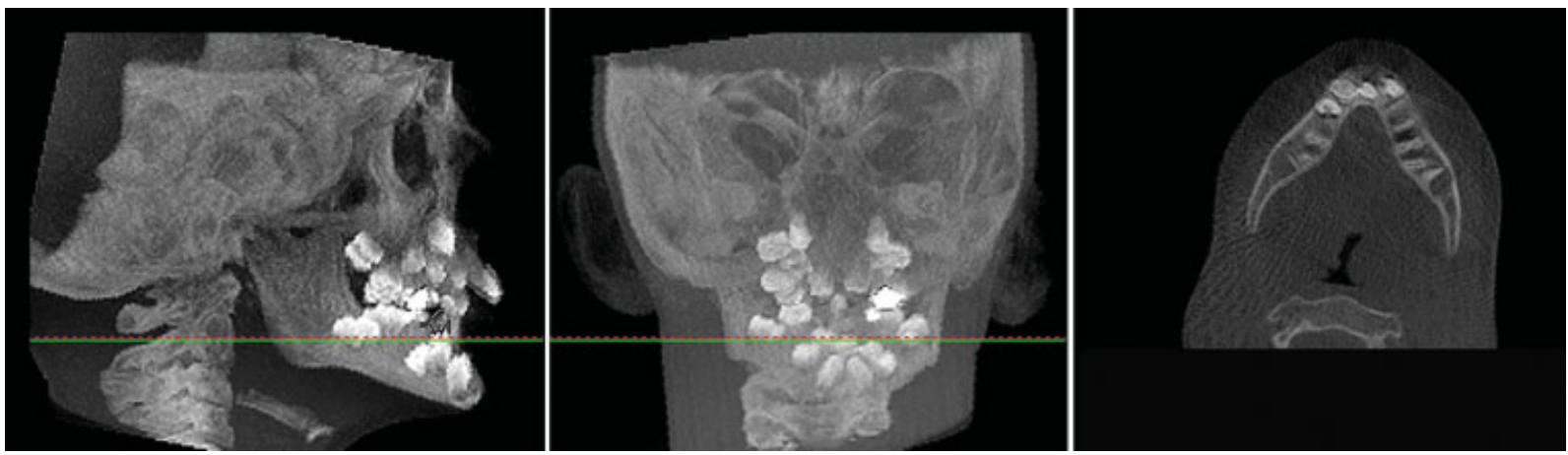

Fig. 6 (A, B, C) Axial coronal slices, mandible. 

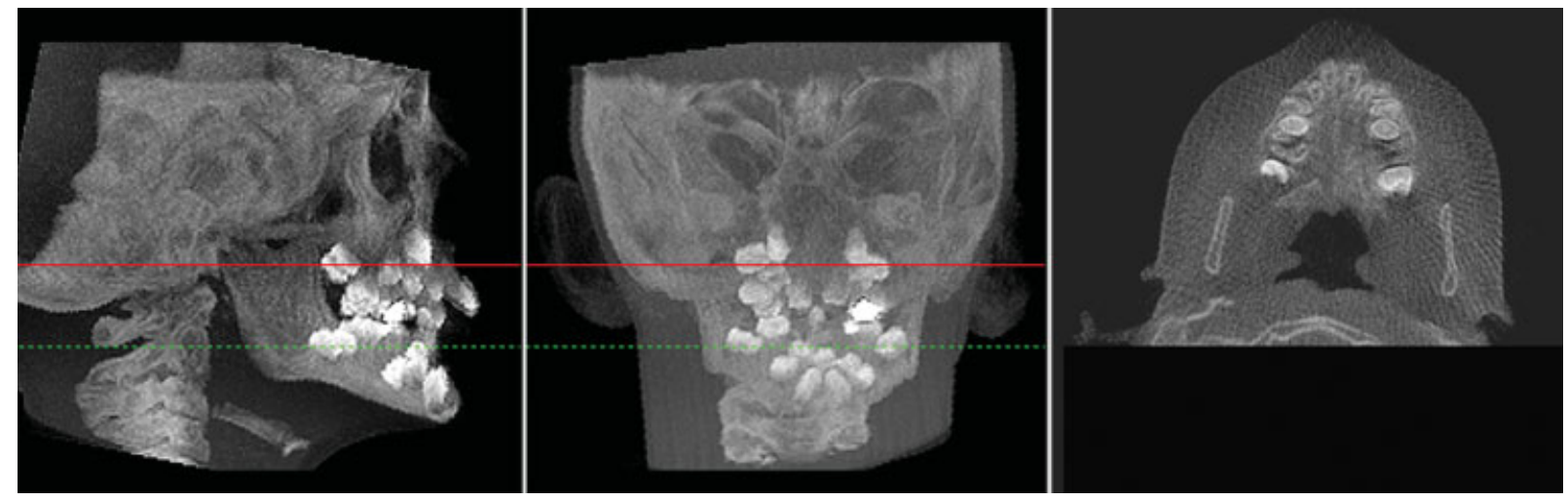

Fig. 7 (A, B, C) Axial coronal slices, maxilla.
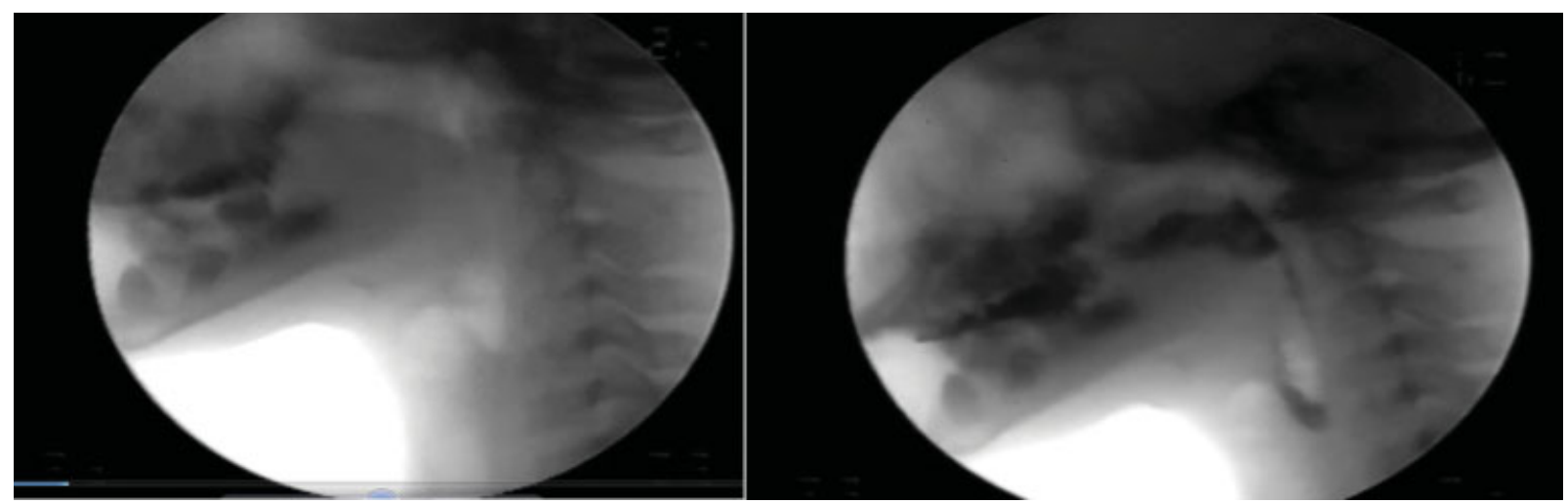

Fig. 8 (A) Swallowing thin liquid, tongue on the floor of the pharynx posterior parade, and (B) swallowing solids, end of the oral procedure, beginning of the pharyngeal phase.

probably reduced natural teeth autolysis, thus resulting in the high number of carious or prematurely lost deciduous teeth.

Skeletally, the necessary transversal gain in the mandible could only be corrected by surgery as the mentonian symphysis usually closes itself before the first year of life. The purpose of orthodontic treatment is to correct malocclusion within the limits imposed by the case. Early assistance to these patients becomes essential as well as myofunctional therapy, ${ }^{12}$ associated with orthodontic intervention, which improves saliva control, leading to higher efficiency in chewing and swallowing all kind of food including solids, with the aid of maneuvers favoring head position.

To better understand the physiology of swallowing, videofluoroscopic assessment was performed, ${ }^{13}$ which initially showed the cavity structures of the mouth palate and pharynx. Swallowing of liquid, pasty, and solid foods were studied. Examination of the floor of the mouth noted movement against the posterior pharyngeal wall to replace the tongue's function in ejection of the bolus. - Fig. 8A shows the movement while swallowing liquids, and - Fig. 8B, while swallowing doughy and solid foods. Despite the absence of the tongue, the patient did not perform postural maneuvers such as head extension to facilitate the ejection of the bolus. Still, it is possible to observe the stasis in the posterior wall of the pharynx, though multiple swallows were necessary for mouth floor cleansing.

Although we found exaggerated movements of the perioral musculature during deglutition, the surface electromyography did not register hyperactivity in the masticatory muscles once they were responsible for moving the mandible, showing reduced participation in deglutition. In opposition to some theorists, the presence of intelligible speech is worth emphasizing, mainly of phonemes and archiphonemes such as $/ \mathrm{r} /$, by means of adjustment of structures like the muscles of oral cavity floor and soft palate during communication. Exaggerated movements of facial muscles during speech production are essential characteristics in cases of aglossia to compensate tongue absence in the production of some phonemes.

\section{Final Comments}

Interdisciplinary and multidisciplinary treatments are necessary and indispensable for patients with aglossia to improve quality of life and to provide adequate conditions so that all resultant myofunctional adaptation may occur in the course of growth and development of craniofacial complex. 


\section{References}

1 Luís R. Dicionário de Termos Técnicos de Medicina e Saúde. 2ª̣ Ed. Rio de Janeiro, Brazil: Editora Guanabara Koogan; 2003

2 Moore KL. Embriologia Clínica. 3르 ed. Rio de Janeiro: Editora Guanabara; 1982:442

3 Salles F, et al. Complete and isolated congenital aglossia: case report and treatment of sequel e using rapid prototyping models. Oral Surg Oral Med Oral Pathol Oral Radiol Endod 2008;105:41-47

4 Kantaputra P, Tanpaiboon P. Thyroid dysfunction in a patient with aglossia. Am J Med Genet A 2003;122A(3): 274-277

5 Felício CM. Desenvolvimento normal das funções Estomatognáticas. In: Ferreira LP, Befi-Lopes DM, Limongi SCO, eds. Tratado de Fonoaudiologia. São Paulo, Brazil: Roca; 2004: 195-211

6 Rasool A, et al. Isolate aglossia in a six year old child presenting with impaired speech: a case report [Internet]. Cases journal; content/pdf/1757-1626-0002-0000007926 [cited 214]. Available at: http://www.casesjournal.com/content/2/1/7926

7 Nevin NC, Burrows D, Allen G, Kernohan DC. Aglossia-adactylia syndrome. J Med Genet 1975;12(1):89-93

8 Kumar P, Chaubey KK. Aglossia: a case report. J Indian Soc Pedod Prev Dent 2007;25(1):46-48

9 Altmann EBC, Khoury RBF, Ramos ALNF. Avaliação fonoaudiológica. In: Fissuras labiopalatinas. $4^{\mathrm{a}}$ ed. Carapicuiba, Brazil: Pro-Fono; 1997:325-366

10 Balters W. Ergebniss der Gesteuerten Selbstheilung von kieferorthopädischen Anomalien. DDZ 1960;15(3):241-248

11 McNamara JA Jr, Brudon WL. Orthodontics and Dentofacial Orthopedics. Ann Arbor, MI: Needhan Press; 2004

12 Hanson ML, Barret RH. Fundamentos de Miologia Orofacial. Rio de Janeiro, Brazil: Enelivros; 1995

13 Silva RG, Furkim AM. Diagnóstico e programas de Reabilitação em Disfagia Orofaríngea Neurogênica. 1st ed. São Paulo, Brazil: Frontis; 1999 